\title{
Modelling of All-Optical Symmetric Mach-Zehnder Switch with Asymmetric Coupler
}

\author{
Nani Fadzlina Naim ${ }^{1}$, Razali Ngah $^{2}$, Teguh Prakoso ${ }^{2}$, \\ Suzi Seroja Sarnin ${ }^{1}$, Husna Abdul Rahman ${ }^{1}$ and Zabih Ghassemlooy ${ }^{3}$ \\ ${ }^{1}$ Centre for Communication Engineering Studies, Faculty of Electrical Engineering, UiTM \\ ${ }^{2}$ Wireless Communication Centre, Faculty of Electrical Engineering, UTM. \\ ${ }^{3}$ Optical Communication Research Lab, School of Engineering \& Technology, Northumbria University, UK \\ nanifadzlina@salam.uitm.edu.my, razalin@fke.utm.my, teguh.prakoso@ieee.org, seroja121@yahoo.com, \\ husna232@salam.uitm.edu.my and fary.ghassemlooy@unn.ac.uk
}

\begin{abstract}
Ultra high-speed optical network is developing rapidly as growing capacity demand in telecommunication system is increasing. In these networks, it is desired to carry out switching, routing and processing in optical domain to avoid bottlenecks of optoelectronic conversions. Optical time-division multiplexing (OTDM) technique is one option to implement all optical networks. It provides a single data stream at a very high rate $(>100 \mathrm{Gbits} / \mathrm{s})$ using a single wavelength. These networks will be based on optical packet switching. The success of these networks depends on how well switching and routing are being done at this very high speed. An all optical switch based on symmetric Mach-Zehnder (SMZ) with asymmetric coupler (60:40) is proposed. Its characteristics and switching window profiles will be investigated. The results show that symmetric Mach-Zehnder (SMZ) with asymmetric coupler gives a better contrast ratio rather than symmetric Mach-Zehnder (SMZ) with normal 50:50 coupler.
\end{abstract}

Keywords: Symmetric Mach-Zehnder (SMZ) switch, Semiconductor Optical Amplifier (SOA), coupler.

\section{Introduction}

Today, optical network is developing rapidly as growing capacity demand in telecommunication system is increasing. Optical transmission systems are typically running at data rates of $2.5 \mathrm{Gbits} / \mathrm{s}$ or $10 \mathrm{Gbits} / \mathrm{s}$ per channel. There are many advantages for designing switching elements using optical components. These advantages include decreased switching time (less than $1 / 10$ of a pico second $\left(10^{12}\right)$ ), less crosstalk and interference, increased reliability, increased fault tolerance, enhanced transmission capacity, economical broadband transport network construction, enhanced crossconnect node throughput, and flexible service provisioning.

When operating at an ultra-high speed (hundreds of $\mathrm{GHz}$ ) requires that the entire signal routing, switching and processing be carried out in the optical domain in order to avoid bottleneck due to the optical to electronic conversion. Most networking equipment today is still based on electronic-signals, meaning that the optical signals have to be converted to electrical ones, to be amplified, regenerated or switched, and then reconverted to optical signals. This is generally referred to as an 'optical -to-electronic-to- optical' $(\mathrm{OEO})$ conversion and is a significant bottleneck in transmission.

The basic premise of optical switching is that by replacing existing electronic network switches with optical ones, the need for OEO conversions is removed. Clearly, the advantages of being able to avoid the OEO conversion stage are significant. First, optical switching should be cheaper, as there is no need for lots of expensive high-speed electronics. Removing this complexity should also make for physically smaller switches. Unfortunately, optical switching technology is still very much in its infancy. There have been numerous proposals as to how to implement light switching between optical fibres, such as semiconductor amplifiers, liquid crystals, holographic crystals and tiny mirrors.

All optical switches such as the terahertz optical asymmetric demultiplexer (TOAD) and the Symmetric Mach Zehnder (SMZ) are the key component for switching and routing due to their ultra fast switching time. Among all optical switches, the SMZ based switches have the most flexibility, a narrow and square switching window, a compact size, thermal stability and low power operation [8]. Symmetric Mach-Zehnder (SMZ) with asymmetric coupler gives a better contrast ratio rather than the typical SMZ switch [3].

\section{Operation Principles}

Figure 1 shows the block diagram of a typical SMZ switch composed of two semiconductor optical amplifiers, one in each arm of the interferometer and a number of 3-dB couplers. Control and data pulses are fed into switch via 3-dB couplers and co- 
propagate within the interferometer. With no control pulse (neither CP1 nor CP2), the SMZ is balanced in such a way that all the data signals emerge at the output port 2. However, with the presence of control signal, a differential phase shift is introduced between the two arms of the interferometer resulting in data pulses being switched to the output port 1 . Note that the time delay, Tdelay between the control pulses and their power intensity determines the nominal width of the switching window.

Figure 2 shows the block diagram of a proposed SMZ switch. The difference of this SMZ switch is that at the end of this switch, a 60:40 coupler is used instead of using a $3 \mathrm{~dB}$ coupler or a 50:50 coupler.

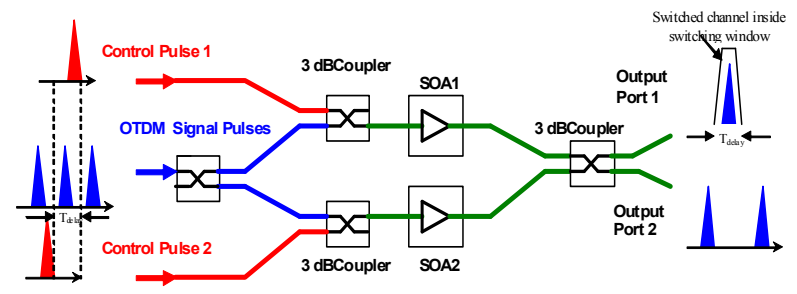

Figure 1: A typical SMZ switch

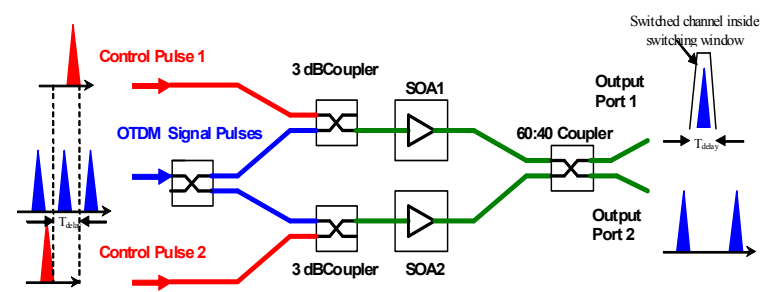

Figure 2: The proposed SMZ switch with asymmetric coupler

The expression for the switching window of the proposed SMZ switch is given by [8]:

$W(t)=\left[(1-\alpha)^{3} G(t)+\alpha^{2}(1-\alpha) \cdot G_{2}(t)-2 \sqrt{(1-\alpha)^{4} \alpha^{2} G(t) \cdot G_{2}(t) \cdot \cos (\phi)}\right](1)$

where $\alpha$ is the coupling ratio of the coupler, G1 and $\mathrm{G} 2$ are the temporal gain profile of the data pulses given as [9]:

$$
\begin{aligned}
& G_{1}(t)=\exp \left[\int_{0}^{L S O A} \Gamma \cdot g\left(z, t+\frac{z}{V_{g}}\right) d z\right] \\
& G_{2}(t)=\exp \left[\int_{0}^{L s o A} \Gamma \cdot g\left(z, t+T_{\text {delay }}+\frac{z}{V_{g}}\right) d z\right]
\end{aligned}
$$

and $\Delta \phi$ is the phase difference between data pulses defined as [9]:

$$
\Delta \phi=-0.5 \alpha_{L E F} \ln \left(G_{1} / G_{2}\right)
$$

$\alpha_{L E F}$ is the linewidth enhancement factor, $\Gamma$ is the confinement factor, $\mathrm{g}$ is the differential gain, $\mathrm{t}$ is the time at which the temporal point of the data signal enters the amplifier, Tdelay the control signal separation, $\mathrm{z} / \mathrm{Vg}$ the time increment in $\mathrm{z}$ direction and $\mathrm{Vg}$ is the group velocity of the control pulse.

\section{Results and Discussions}

The modelling of the SMZ switch is done by using Matlab. The gain profiles of SOA and the switching window profiles of the SMZ switch is observed and compared between a typical SMZ switch and the proposed SMZ switch. The contrast ratio is determined from the switching window profile. The contrast ratio is defined as:

$$
\text { Contrast Ratio }(d B)=\left(\begin{array}{l}
\text { Highest } \text { power } \\
\text { of } \text { wanted signal }
\end{array}\right)-\left(\begin{array}{l}
\text { Lowest } \text { power of } \\
\text { wanted signal }
\end{array}\right)
$$

Figure 3 shows the gain profiles of the SOA1 (G1, gain at the upper arm of SMZ switch) and SOA2 (G2, gain at the lower arm of the SMZ switch). Since the time delay between the control signals is $5 \mathrm{ps}$, then identical gain profiles of G1 and G2 will be obtained. However the gain profile of SOA2, G2 is delayed by $5 \mathrm{ps}$ (the same value as the time delay between the control pulse).

Using the same parameter as before, switching window profile as Figure 4 is obtained. It can be seen that, whenever the time delay between the control signals is 5ps, a switching window with FWHM equal to $5 \mathrm{ps}$ is achieved. The switching window has a square and symmetrical shape due to the propagating data and control signals that pass through the SMZ switch. A square shape of switching window profile is very crucial and desirable in OTDM network where there is need to extract or multiplex the target channel from the OTDM signal with little crosstalk.

Using the same parameter, switching window profile for the proposed SMZ switch is obtained as in Figure 5. The contrast ratio for the proposed SMZ switch is higher, $7 \times 10^{-3}$ as compared to the typical SMZ switch with contrast ratio $7 \times 10^{-4}$.

Figure 6 shows the switching window profiles of SMZ switch (using 50:50 coupler) for different time delay in $1 \mathrm{ps}$ step. From here, it can be seen that there is no increase in SMZ gain for time delay more than 5 ps.

Using the same parameters, the switching window profiles for different time delay in $1 \mathrm{ps}$ step for the proposed SMZ switch is obtained as in Figure 7. It is found that in term of contrast ratio, the proposed SMZ switch has better contrast ratio. However, in term of the shape, the proposed SMZ 
switch has unsymmetrical shape of switching window.

Figure 8 is the experimental result of switching window profile that has been done by Toliver [1] From the results using experimental and numerical calculation, the switching window is similar in term of the shape. This experimental result has a greater rise time as the time delay is in 1.6ps step.

Figure 9 shows the comparison in SMZ switching window between the two types of SMZ switch. By applying bias current more than 0.8Amp with linewidth enhancement factor more than 5 will result with no increase in contrast ratio and poor flat top. Therefore, if high bias $\operatorname{current}(\geq 0.8 \mathrm{~A})$ is applied, low linewidth enhancement $(<5)$ must be used and vice versa.

Figure 10 shows the comparison of contrast ratio for different SOA length between the 2 types of SMZ switch. From the graph, we can see that a higher contrast ratio is achieved by using SMZ switch with 60:40 coupler. A practical value of SOA length is in the range of $1 \times 10^{-4}$ to $3 \times 10^{-4} \mathrm{~m}$.

Figure 11 shows the comparison of the contrast ratio for different linewidth enhancement factor between the 2 types of SMZ switch. The optimum and practical linewidth enhancement for both types of SMZ switch is in the range from 1 to 7 (for bias current $=0.6 \mathrm{~A}$ ). SMZ switch using 60:40 coupler has significant increase in contrast ratio for linewidth enhancement less than 6 .

From Figure 12, a higher contrast ratio is achieved by using the proposed SMZ switch. Both SMZ switches show that there is no increase in contrast ratio for time delay more than $5 \mathrm{ps}$.

Figure 13 shows the comparison of full wave half magnitude (FWHM) for different time delay between the 2 types of SMZ switches. From the graph, it can be seen that there is a slight different between the 2 types of SMZ switches. FWHM increase almost linearly as the time delay increase for both types of switches.

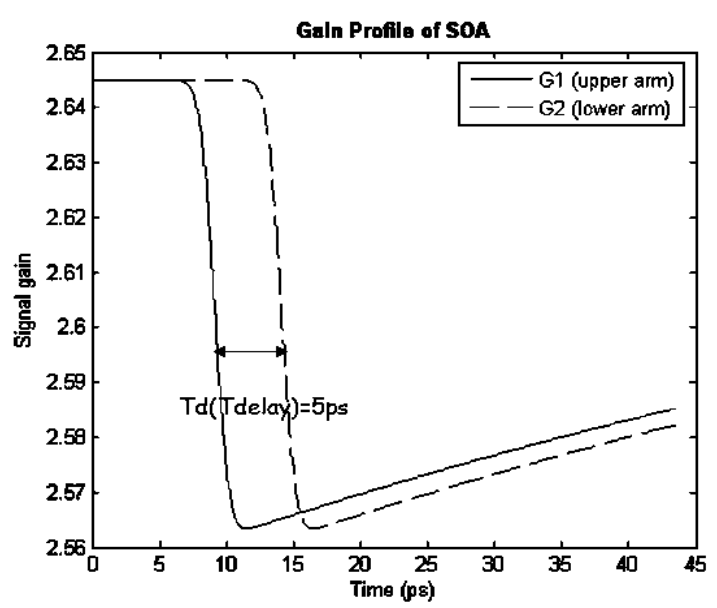

Figure 3: Gain profiles of SOA
Table 1: SMZ switch parameters for Figure 3

\begin{tabular}{|l|c|}
\hline \multicolumn{1}{|c|}{ SMZ switch parameters } & Value \\
\hline Injected current & $0.3 \mathrm{~A}$ \\
\hline Group velocity & $3 \mathrm{e} 8 / 3.5 \mathrm{~m} / \mathrm{s}$ \\
\hline Photon charge, $\mathrm{q}$ & $1.602 \times 10^{-19}$ \\
\hline Spontaneous emission time & $100 \times 10^{-12} \mathrm{~s}$ \\
\hline Confinement factor & 0.15 \\
\hline Linewidth enhancement factor & 4 \\
\hline Photon energy & $0.8 \times \mathrm{q}$ \\
\hline Gain coefficient & $2 \mathrm{e}-20$ \\
\hline Transparent carrier density & $1 \times 10^{24} \mathrm{~m}^{-3}$ \\
\hline Area of SOA & $3 \times 10^{-13} \mathrm{~m}$ \\
\hline Electrical field & $2.598 \times 10^{-13} \mathrm{~V} / \mathrm{m}$ \\
\hline Length of SOA & $2.5 \times 10^{-4} \mathrm{~m}$ \\
\hline Total no. of segment & 50 \\
\hline Length interval & $2.5 \times 10^{-4} / 50$ \\
\hline Time increment for calculation & $5.83 \times 10^{-14}$ \\
\hline Control pulse width & $2 \times 10^{-12} \mathrm{~m}$ \\
\hline Control pulse peak power & $1 \mathrm{~W}$ \\
\hline Time delay & $5 \times 10^{-12} \mathrm{~s}$ \\
\hline
\end{tabular}

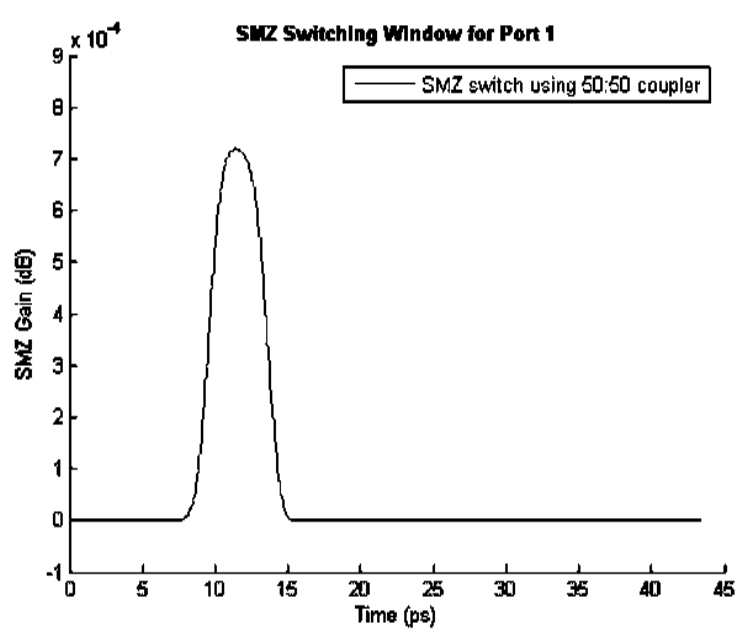

Figure 4: Switching window profile for typical SMZ switch

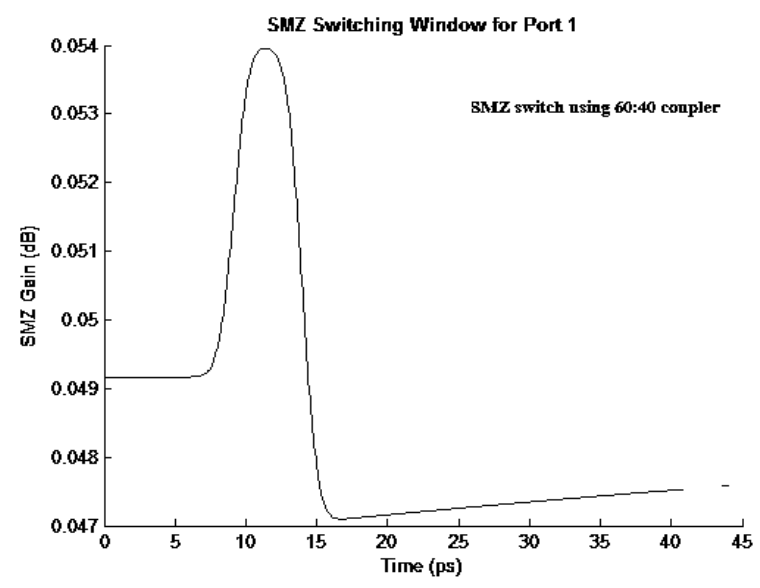

Figure 5: Switching window profile for the proposed SMZ switch 


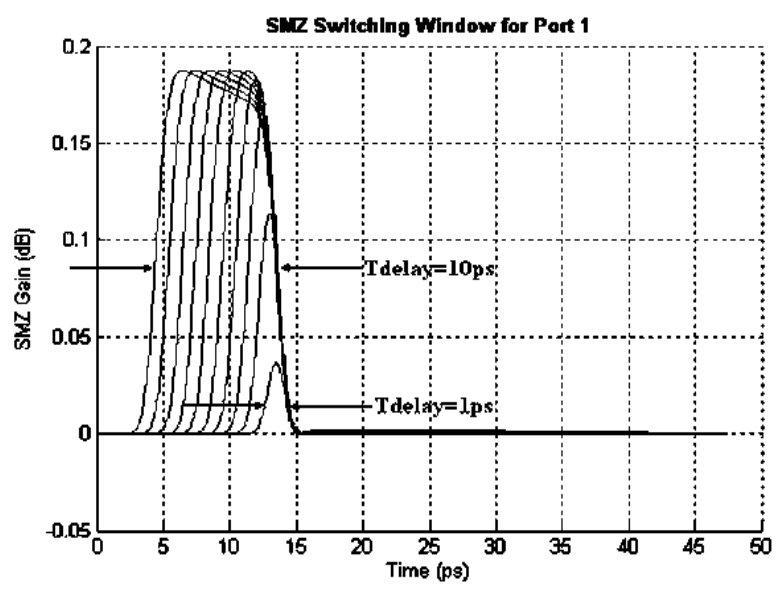

Figure 6: Switching window profiles of typical SMZ switch for different time delay in 1 ps step

Table 2 : SMZ switch parameters for Figure 6

\begin{tabular}{|l|c|}
\hline \multicolumn{1}{|c|}{ SMZ switch parameters } & Value \\
\hline Injected current & $0.6 \mathrm{~A}$ \\
\hline Group velocity & $3 \mathrm{e} 8 / 3.5 \mathrm{~m} / \mathrm{s}$ \\
\hline Photon charge, $\mathrm{q}$ & $1.602 \times 10^{-19}$ \\
\hline Spontaneous emission time & $100 \times 10^{-12} \mathrm{~s}$ \\
\hline Confinement factor & 0.15 \\
\hline Linewidth enhancement factor & 3 \\
\hline Photon energy & $0.8 \times \mathrm{q}$ \\
\hline Gain coefficient & $2 \times 10^{-20}$ \\
\hline Transparent carrier density & $1 \times 10^{24} \mathrm{~m}^{-3}$ \\
\hline Area of SOA & $3 \times 10^{-13} \mathrm{~m}$ \\
\hline Electrical field & $2.598 \times 10^{-13} \mathrm{~V} / \mathrm{m}$ \\
\hline Length of SOA & $3 \times 10^{-4} \mathrm{~m}$ \\
\hline Total no. of segment & 50 \\
\hline Length interval & $3 \times 10^{-4} / 50$ \\
\hline Time increment for calculation & $7 \times 10^{-14}$ \\
\hline Control pulse width & $2 \times 10^{-12} \mathrm{~m}$ \\
\hline Control pulse peak power & $1 \mathrm{~W}$ \\
\hline Time delay & Varies \\
\hline
\end{tabular}

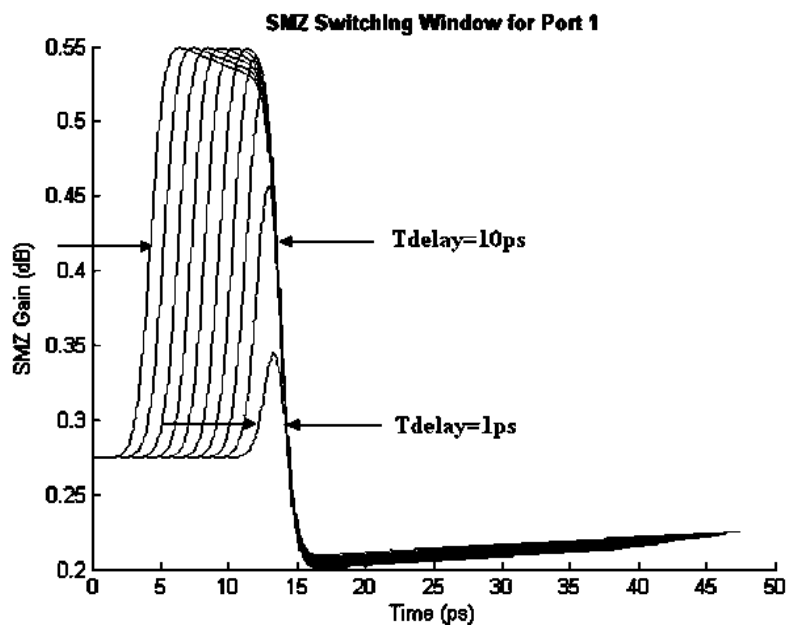

Figure 7: Switching window profiles of proposed SMZ switch for different time delay in 1 ps step

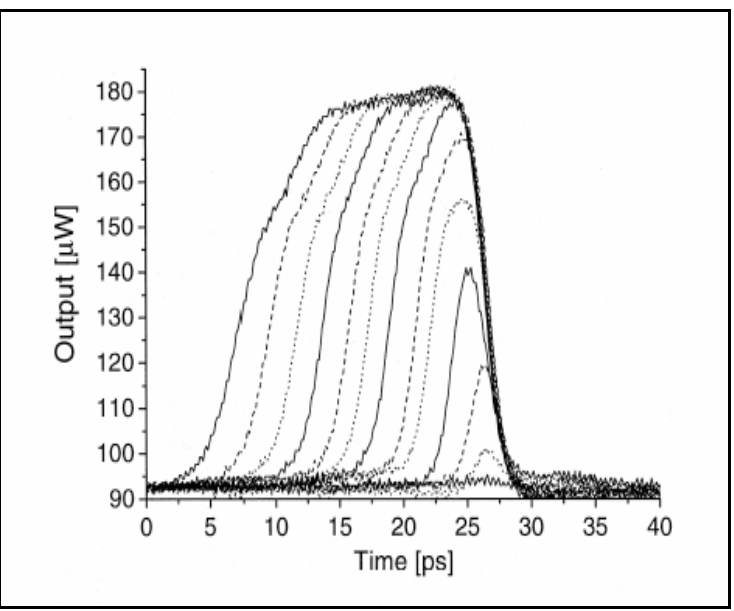

Figure 8: Switching window profiles for different time delay in 1.6ps step [1]

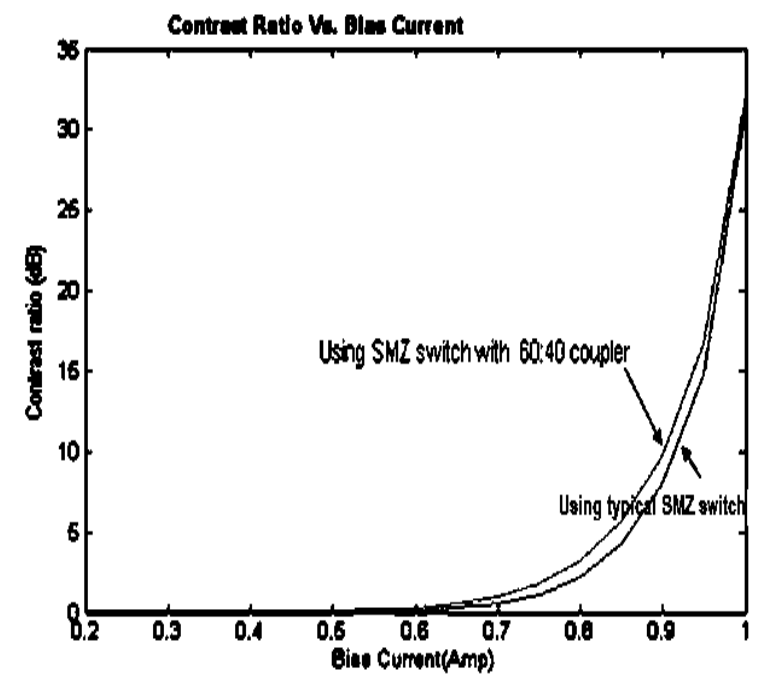

Figure 9: Contrast Ratio versus Bias current

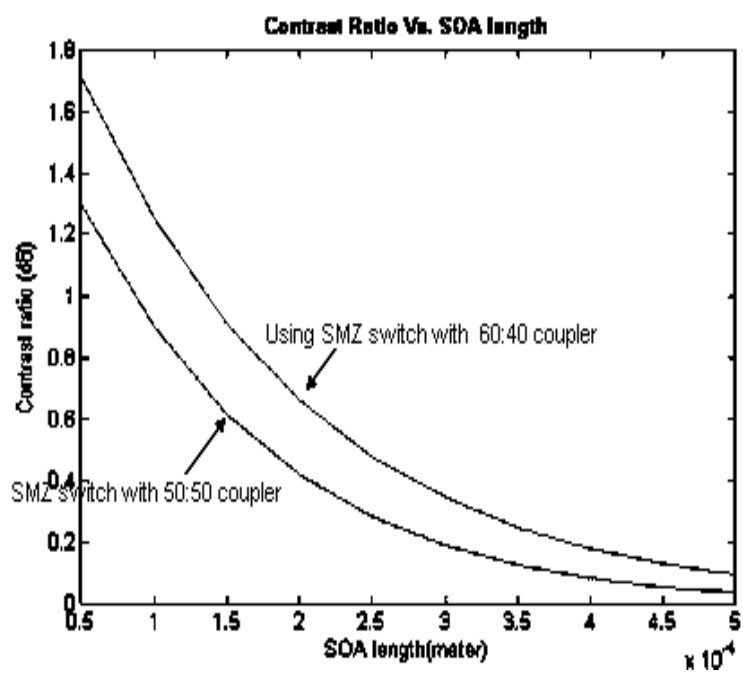

Figure 10: Contrast ratio versus SOA length 


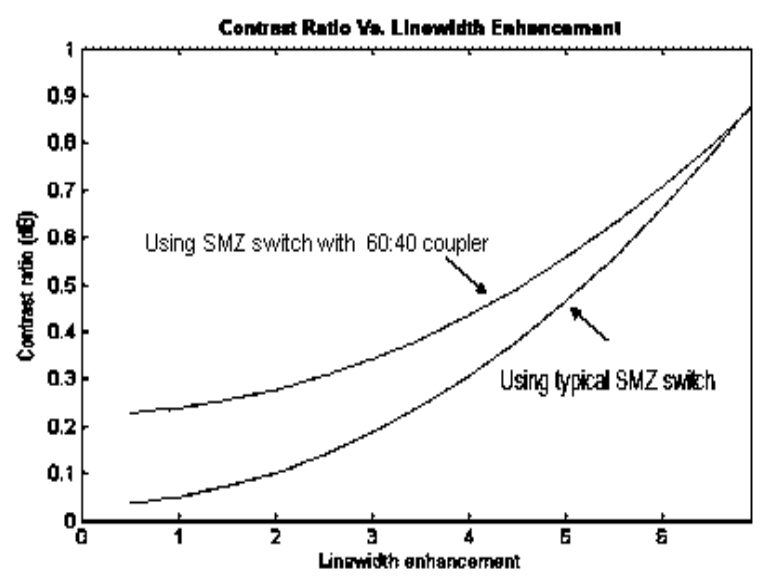

Figure 11: Contrast ratio versus Linewidth Enhancement Factor

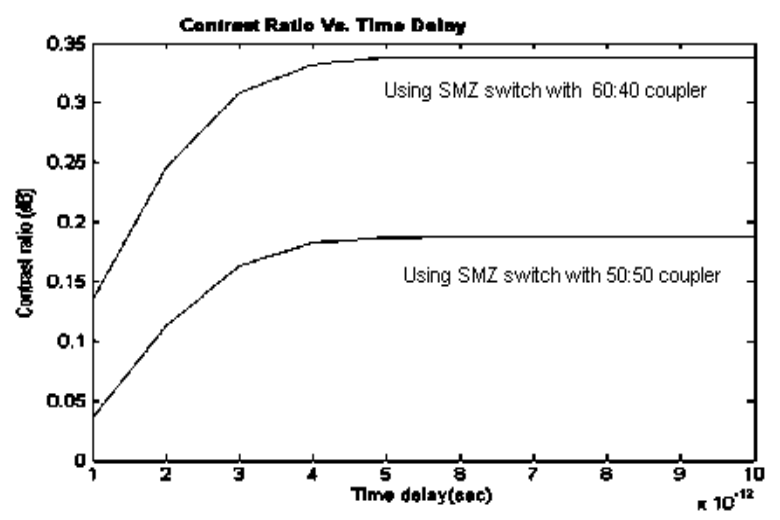

Figure 12: Contrast ratio versus Time delay

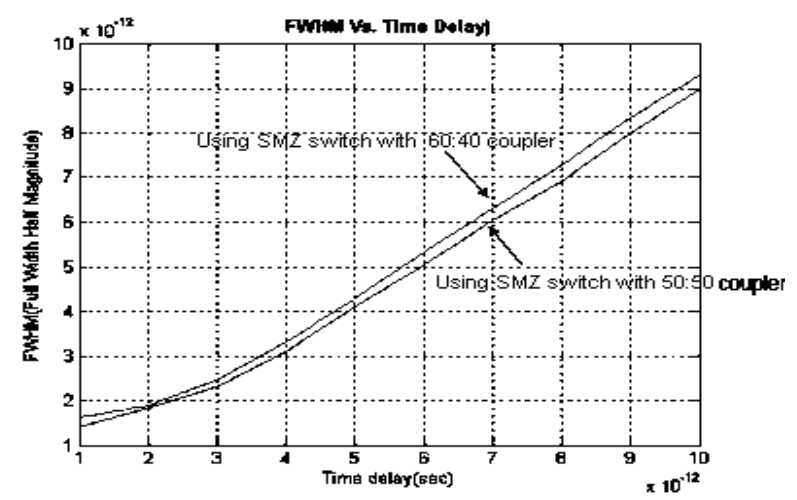

Figure 13: FWHM versus Time delay

Table 3: Data and Control signal Parameters

\begin{tabular}{|c|c|}
\hline Data and control signals parameters & Value \\
\hline Wavelengths & $1550 \mathrm{~nm}$ \\
\hline Signal FWHM & $2 \mathrm{ps}$ \\
\hline Control signal peak power & $2.5 \mathrm{~W}$ \\
\hline Data signal peak power & $2.5 \mu \mathrm{W}$ \\
\hline
\end{tabular}

\section{Summary}

Modelling of all optical switch based on Symmetric Mach-Zehnder with asymmetric coupler is presented in this paper. The comparison between SMZ switch with symmetric coupler and SMZ switch with asymmetric coupler is investigated. The results show that SMZ switch with asymmetric coupler is better than the typical SMZ switch in terms of its contrast ratio.

\section{References}

[1] P. Toliver, I. Glesk, P.R. Prucnal, “All-optical clock and data separation technique for asynchronous packet-switched optical time division-multiplexed networks," Optical Commun.,vol. 173, pp. 101-106, 2000.

[2] I. Glesk, R. J. Runser, P. R. Prucnal, “New Generation of Devices For All-Optical Communication," Acta physica slovaca, vol. 51, No. 2, 151 - 162, April 2001.

[3] Jurg Leuthold,"Advanced Indium-Phosphide waveguide MZI all optical switches and wavelength converters," PhD Dissertation, Hartung-Gorre Verlag Konstanz, Germany, 1999.

[4] X. Ma, G. S. Kuo, "Optical Switching Technology comparison: optical MEMS vs. other technologies," IEEE Optic Commun., vol. 41, pp. S16-S23, 2003.

[5] S.Nakamura et al., "Experimental investigation on high-speed switching characteristics of a novel symmetric Mach-Zehnder all optical switch," Appl. Phy. Lett., vol. 65, pp. 283-285, 1994.

[6] R. Ngah, Z. Ghassemlooy, and G. Swift, "Comparison of Interferometric all optical switches for router applications in OTDM systems" 4th Annual Postgraduate Symposium on Convergence of Telecommunications, Networking and Broadcasting, Liverpool, U.K, 16-17 June 2003, pp. 81-85.

[7] T.Ohara et al., "160 Gbps OTDM transmission using integrated all-optical MUX/DEMUX with all channel modulation and demultiplexing," IEEE Photon. Tech. Lett., vol.16, pp.650-652 2004.

[8] R. P. Schreieck, M. H. Kwakernaak, H. Jackel, and H. Melchior, "All optical Switching at multi-100-Gb/s data rates with Mach-Zehnder interferometer switches," IEEE Quan. Elec., vol. 38, pp.1053-1061, 2002.

[9] Eiselt, M., Pieper, W., and Weber, H.G.:SLALOM: semiconductor laser amplifier in a loop mirror', J.Lightwave Technol., 1995, 13, (10), pp. 2099-2112

[10] N.S. Patel, K. A. Rauschenbach, and K.L.Hall, "40 Gbps demultiplexing using an Ultrafast Nonlinear Interferometer," IEEE Photon. Tech.Lett., vol. 8, pp. 1695-1697, 1996.

[11] P.R. Prucnal, R. J. Runser, and M. H. Singer "Optical Switching techniques for the next generation Internet: a competitive study," White Paper, Ultrafast Optical Systems, pp. 1-14, 2000. 